\title{
Staging of T1 esophageal adenocarcinoma with volumetric laser endomicroscopy: a feasibility study
}

\section{다)(요 $\odot$}

Authors

Allon Kahn', Amrit K. Kamboj' ${ }^{1}$ Prasuna Muppa ${ }^{3}$, Tarek Sawas ${ }^{4}$, Lori S. Lutzke, Matthew R. Buras ${ }^{5}$, Michael A. Golafshar ${ }^{5}$, David A. Katzka ${ }^{4}$, Prasad G. Iyer ${ }^{4}$, Thomas C. Smyrk ${ }^{4}$, Kenneth K. Wang ${ }^{4}$, Cadman L. Leggett ${ }^{4}$

Institutions

1 Division of Gastroenterology and Hepatology, Mayo Clinic, Scottsdale, Arizona, United States

2 Department of Internal Medicine, Mayo Clinic, Rochester, Minnesota, United States

3 Department of Laboratory Medicine and Pathology, Mayo Clinic, Rochester, Minnesota, United States

4 Division of Gastroenterology and Hepatology, Mayo Clinic, Rochester, Minnesota, United States

5 Division of Health Sciences Research, Mayo Clinic, Scottsdale, Arizona, United States

submitted 10.8.2018

accepted after revision 26.11.2018

\author{
Bibliography \\ DOI https://doi.org/10.1055/a-0838-5326 | \\ Endoscopy International Open 2019; 07: E462-E470 \\ (c) Georg Thieme Verlag KG Stuttgart · New York \\ ISSN 2364-3722
}

Corresponding author

Cadman L. Leggett, M.D., Division of Gastroenterology and Hepatology, Mayo Clinic, 200 1st, Street SW, Rochester, MN 55905

Fax: +1-480-301-8673

Leggett.Cadman@mayo.edu

Supplemental Table 1

Online content viewable at:

https://doi.org/10.1055/a-0838-5326

\section{ABSTRACT}

Background and study aims Precise staging in T1 esophageal adenocarcinoma (EAC) is critical in determining candidacy for curative endoscopic resection. High-frequency endoscopic ultrasound (EUS) has demonstrated suboptimal accuracy in T1 EAC staging due to insufficient spatial resolution. Volumetric laser endomicroscopy (VLE) allows for high-resolution wide-field visualization of the esophageal microstructure. We aimed to investigate the role of VLE in staging T1 EAC.

Patients and methods Patients undergoing endoscopic mucosal resection (EMR) were prospectively enrolled and only T1 EAC cases were included. EMR specimens were imaged using second-generation VLE immediately after resection. VLE images were analyzed for signal intensity by depth and signal attenuation $(\mathrm{dB} / \mathrm{mm})$ in both cross-sectional and en-face orientation. A decision tree model was constructed to combine measured VLE parameters and delineate diagnostic thresholds.

Results Thirty EMR scans were obtained - 15 T1a specimens from 9 patients and 15 T1b specimens from 11 patients. T1b specimen VLE scans exhibited higher signal intensity $(P<0.0001)$ and higher signal attenuation compared to T1a specimens $(P=0.03)$. A combination of signal attenuation and signal intensity at $150 \mu \mathrm{m}$ depth yielded optimal diagnostic thresholds and an area under the curve (AUC) of 0.77 . VLE signal attenuation was significantly associated with grade of differentiation, irrespective of EAC stage.

Conclusions VLE signal intensity and signal attenuation are quantitatively distinct in T1a and T1b EAC and associated with grade of differentiation. This is the first study examining the role of VLE for staging of T1 EAC and demonstrates promising diagnostic performance. With further in vivo validation, VLE may serve a role in staging superficial EAC.

\section{Introduction}

Esophageal adenocarcinoma (EAC) is a malignancy with increasing incidence and poor overall 5-year survival [1,2]. Historically, esophagectomy has been the treatment of choice for early stage EAC; however, endoscopic therapy is rapidly gaining acceptance as patients seek to avoid surgical complications [3, 4]. Studies comparing endoscopic mucosal resection (EMR) to esophagectomy for T1 stage EAC demonstrate comparable 
long-term outcomes with fewer complications [5, 6]. However, accurate staging is critical to selecting the appropriate therapeutic approach, as T1b EAC is associated with a higher rate of lymph node metastasis (LNM). Consequently, T1b EAC is generally managed surgically, while T1a EAC can be endoscopically resected with curative intent [7].

EMR currently serves as the standard endoscopic method for staging T1 EAC, as it assesses depth of invasion more accurately than mucosal biopsies and provides other prognostic determinants, including grade of differentiation and lymphovascular invasion [8,9]. However, EMR is invasive and associated with a small but non-negligible risk for complications, including bleeding, perforation, and stricture formation [10]. As a result, endoscopic imaging has been studied in a putative adjunctive role in guiding endoscopic resection. High-frequency endoscopic ultrasound (EUS) lacks the depth resolution to differentiate T1a from T1b disease, demonstrating staging accuracy of only $65 \%$ to $85 \%$. Rather, EUS is best utilized to evaluate for LNM in cases where EMR has already established T1b disease. Thus, current society guidelines suggest that EUS not be utilized routinely prior to EMR in staging early or T1 EAC [11].

Optical coherence tomography (OCT) is a novel technology used for esophageal imaging in which an infrared laser is aimed at the tissue of interest and each cellular structure contained within exhibits differential absorption and reflection. The reflected light is detected as "back-scattering" and generates a digital signal that is reconstructed into a visible image. The principal advantage of OCT is its spatial resolution of 7 to $20 \mu \mathrm{m}$, which vastly exceeds that of EUS, and its ability to perform wide-field imaging. Volumetric laser endomicroscopy (VLE) is a second-generation application of endoscopic probebased OCT in which a probe centered within a balloon undergoes automated helical pullback to image a $6-\mathrm{cm}$ segment of the esophagus in real-time, allowing for rapid wide-field imaging of the esophageal microstructure to a depth of $\sim 3 \mathrm{~mm}$ and an axial resolution of $7 \mu \mathrm{m}$.

Existing studies of VLE have consistently demonstrated that EAC is characterized by a lack of mucosal layering, a homogenous subsurface architecture, and increased surface signal intensity $[12,13]$. Although a growing body of literature suggests that VLE accurately identifies dysplasia in Barrett's esophagus [14], the role of VLE for staging T1 EAC has not been examined. Thus, we sought to identify quantitative VLE image characteristics that would distinguish T1a from T1b EAC and predict other prognostic determinants.

\section{Patients and methods}

\section{Patient selection and endoscopic resection}

Patients referred for evaluation at a tertiary-care Barrett's esophagus (BE) unit at the Mayo Clinic in Rochester, Minnesota, United States were prospectively consented and enrolled from 2014 to 2016. EMR was conducted as clinically indicated. EMR specimens were obtained by a single endoscopist (K.K.W.) with extensive experience in the endoscopic management of EAC, utilizing the cap-snare (Olympus USA, Center Valley, Pennsylvania, United States) or band-ligation technique (Duette, Wilson-
Cook Medical, Winston-Salem, North Carolina, United States). Inclusion criteria consisted of a pathologic diagnosis of EAC staged as T1 by an expert gastrointestinal pathologist (T.C.S). T1b EAC was defined histologically by submucosal invasion, with T1a confined to the mucosal layer. For the purposes of this study, when submucosal tissue was absent and the deep margin was positive, the specimen was classified as T1b given the associated higher risk of LNM compared to specimens with a negative deep margin. Analyses were carried out on a perspecimen basis. This protocol was approved by the Institutional Review Board.

\section{VLE imaging and scan selection}

EMR specimens were imaged using second-generation Nvision VLE (NinePoint Medical, Bedford, MA) immediately after resection, as previously described [13]. Briefly, EMR specimens were rinsed with phosphate-buffered saline and placed in a custombuilt holding device to minimize movement during imaging. Specimens were then submitted for histopathologic evaluation in the standard method.

\section{Ex-vivo EMR image análisis}

All VLE image analysis was conducted in the Fiji distribution of Image] [15]. For image analysis, signal intensity refers to the "brightness" of a pixel on a 0-255 grayscale, while signal attenuation refers to the decrease in signal intensity over distance (i. e. depth) and is quantified in units of $\mathrm{dB} / \mathrm{mm}$. Image analysis methods are depicted in Fig 1 .

\section{Cross-sectional signal intensity analysis}

To analyze the individual signal intensity pattern as a function of depth for each EMR, VLE scans were converted into a stack of 8-bit grayscale image files ( $>$ Fig. 1 a). To account for the curvilinear contour of the balloon surface, a custom plugin was applied to the image stack, wherein the contour of the balloon was flattened and pegged to the edge of the image ( Fig.1b). Thus, the two-dimensional relationship between pixels was preserved. A region of interest (ROI) was created around the EMR specimen to avoid measurement of signal from surrounding artifact. The PlotProfile plugin was then utilized to measure average signal intensity across the entire EMR specimen at each pixel of depth ( $\triangleright$ Fig. 1c).

In an effort to provide complete resection, EMR specimens frequently contain adjacent noncancerous tissue. Because the signal intensity analysis described above inherently includes all tissue types contained in the EMR, we endeavored to identify and analyze only the region of EAC within the specimen in an en-face orientation. EAC uniformly exhibits a higher VLE surface signal intensity and signal attenuation than normal squamous epithelium and can be reliably distinguished by locating the area of highest attenuation [13].

\section{En-face signal intensity analysis}

VLE scans were processed using en-face reconstruction software (Nine Point Medical, Bedford, Massachusetts, United States) that mapped the signal intensity across each specimen at specified levels of depth. In contrast to the cross-sectional 

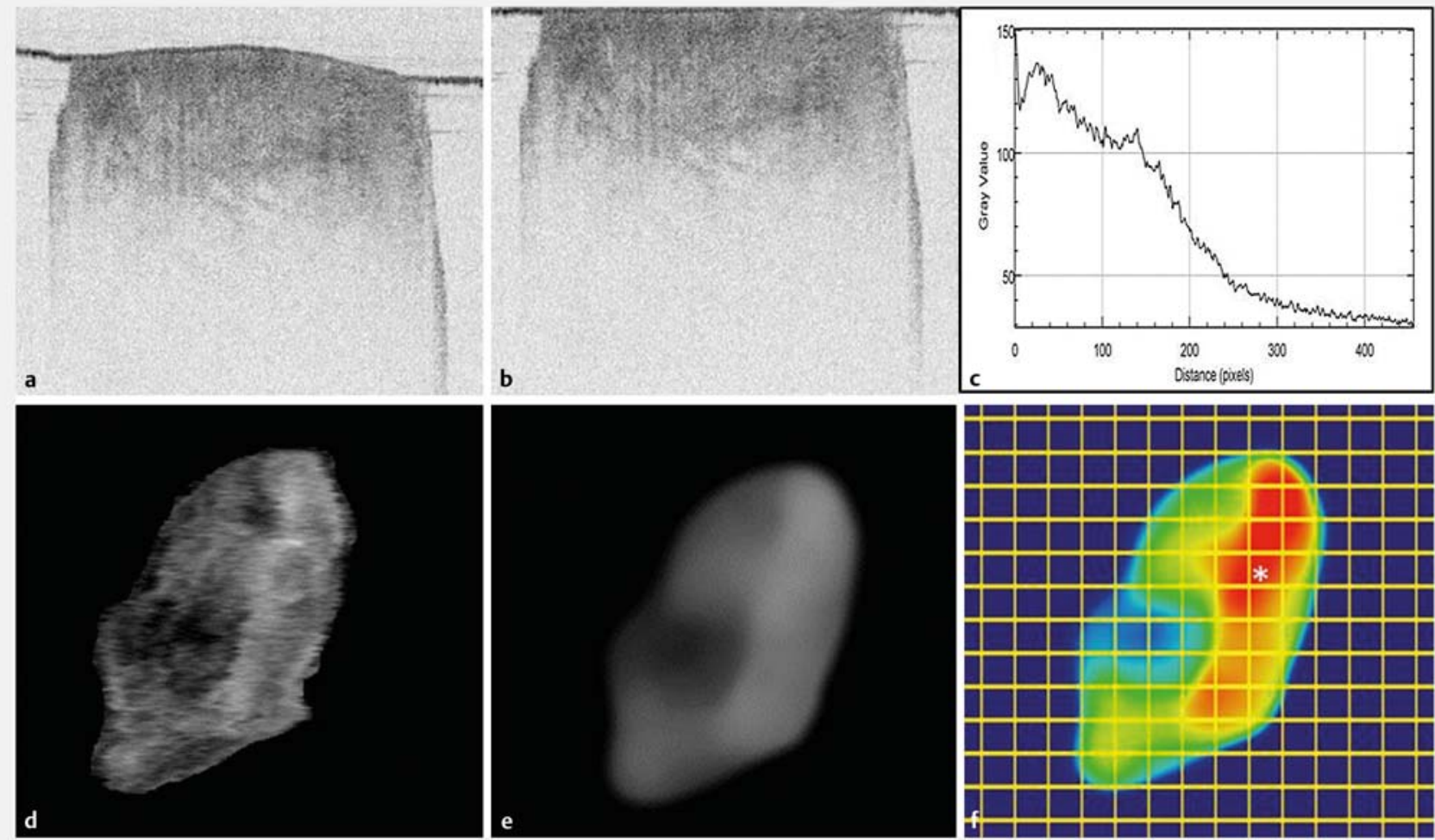

- Fig. 1 Image analysis methods. Cross sectional analysis is depicted in panes a-c, en-face analysis in panes $\mathbf{d}-\mathbf{f}$. a A single ex-vivo crosssectional EMR image from within a stack of image slices through the specimen. $\mathbf{b}$ Each image was flattened by an algorithm, with preservation of the two-dimensional pixel relationship. $\mathbf{c}$ The signal plot profile for the image in pane $\mathbf{b}$. Y-axis is the absolute signal intensity and $\mathbf{x}$-axis is depth in pixels. At each pixel of depth (i. e. from the balloon surface), signal intensity was averaged across the specimen (left-to-right). d En-face reconstruction of the EMR. Pixel intensity in this image represents the slope of signal attenuation $(\mathrm{dB} / \mathrm{mm})$. e The EMR image was filtered through median and mean filters and a $20 \times 20$ pixel grid overlayed, $\mathbf{f}$ with the point of greatest signal attenuation (white asterisk) delineating the region of cancer.

approach, this would be akin to looking down on the resected specimen from above. Using the results of cross-sectional analysis, we identified the depth of maximal signal intensity difference between T1a and T1b specimens. Using the en-face image at this depth, the area of the EMR was manually traced to create a ROI and avoid measurement of surrounding structures (e. g. cork, fluid, etc.) ( $\triangleright$ Fig.1d). The en-face image was processed through a $20 \times 20$ pixel median filter and mean filter with 5 pixel radius ( $\triangleright$ Fig. 1e). A grid of $20 \times 20$ pixel squares was then overlaid on the image. The mean signal intensity in each of the squares was calculated and the value and location of the maximum signal intensity was recorded ( $\mathbf{F i g . 1 f}$ ).

\section{En-face signal attenuation analysis}

A similar ROI analysis was conducted using en-face reconstructions that mapped the signal attenuation pattern across each specimen. Mean signal attenuation in each of the squares was calculated and the value and location of the maximum signal attenuation was recorded.

\section{Pathologic correlation}

All EMR specimens were re-examined by a gastrointestinal pathologist blinded to VLE analysis data. For the purposes of statistical analysis, grade of differentiation was dichotomized as well-differentiated and poor/moderately differentiated.

\section{Statistical analysis}

Statistical analysis was conducted in JMP version 10, RStudio ${ }^{5}$ version 1.0.153, and $R$ version 3.4.2. Descriptive statistics were recorded as mean \pm standard deviation (SD) and median \pm interquartile range (IQR). Average signal intensity measurements at each pixel of depth and maximum mean signal attenuation values were averaged for each EAC subtype. Mean signal intensity values were compared between T1a and T1b groups at each pixel of depth using the student's $t$-test. Mean signal attenuation values were compared between T1a and T1b groups using the Mann-Whitney-U test.

A logistic regression model was implemented to determine the optimal signal attenuation threshold for differentiating T1a from T1b EAC. Logistic regression models were then constructed to analyze whether signal attenuation slope was predictive of both EAC stage and grade of differentiation. Finally, a decision-tree model was constructed, incorporating signal at- 


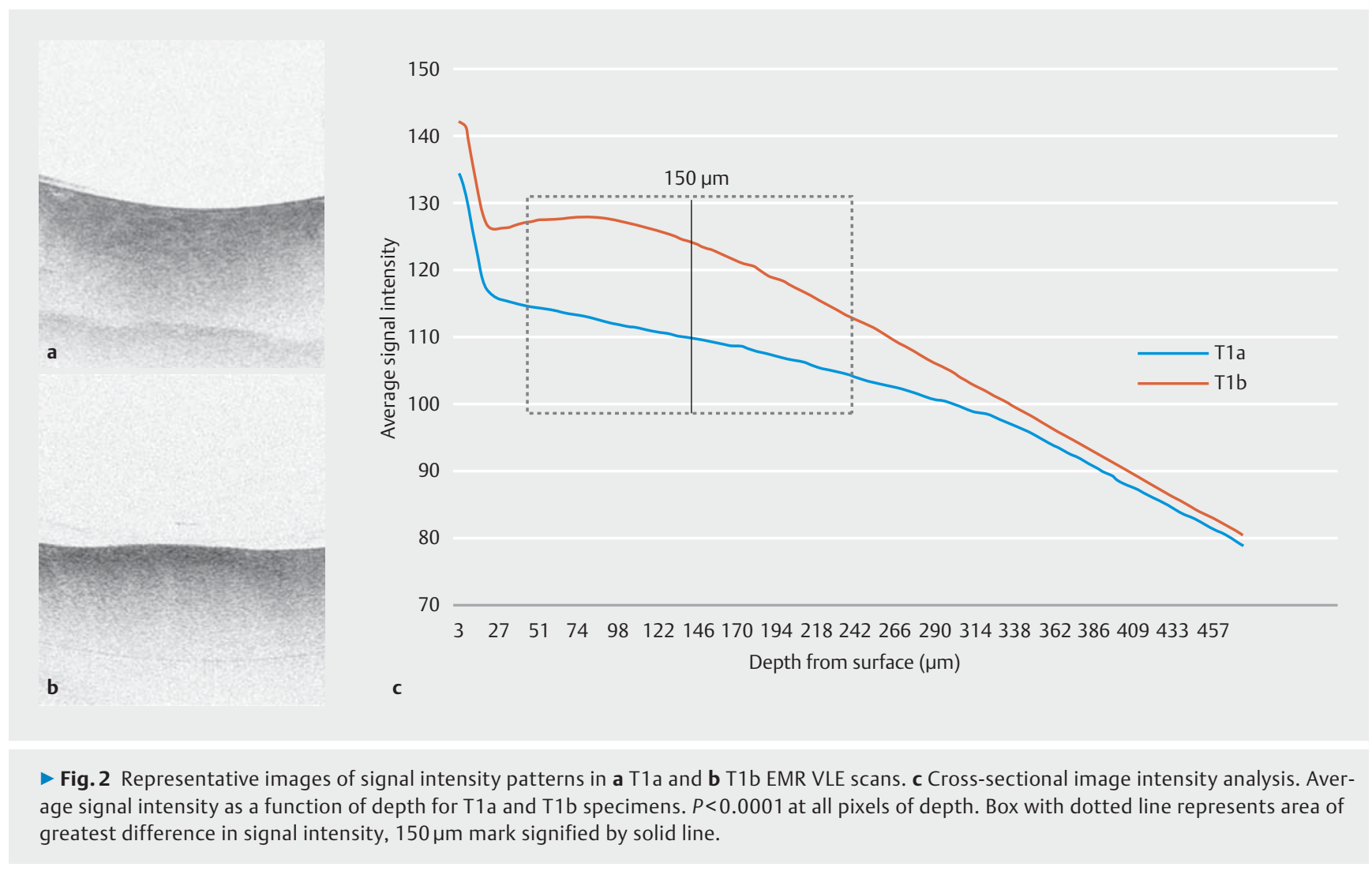

tenuation slope, average and maximum signal intensity at each level of specified depth in the ROI analysis above, and histologic grade. A bootstrap adjusted area under the curve (AUC) was estimated for each model using 1000 bootstrap samples. All statistical tests were two-tailed and a $P$ value $<0.05$ was used to denote statistical significance.

\section{Results}

\section{Patients}

A total of 30 EMR specimens were obtained and imaged with VLE. 15 T1a specimens (mean size $10.9 \mathrm{~mm} \pm 1.9$ ) were obtained from nine patients and $15 \mathrm{~T} 1 \mathrm{~b}$ specimens (mean size $11.5 \mathrm{~mm} \pm 3.6$ ) from nine patients. Four patients underwent resection of a gastroesophageal junction mass without evidence of surrounding BE. Five patients underwent resection of a nodule or mass occurring in the setting of short-segment BE. The remaining seven patients underwent resection of a nodule or mass occurring in the setting of long-segment BE (median $10 \mathrm{~cm}$ ). In two instances, separate resection specimens from the same patient contained both T1a and T1b and were analyzed as such. All lesions were Paris classification Ila, Ila + Ilc, or Is. A single lesion had a broad-based pedical and was thus categorized as Paris Ip. A full description of endoscopic, clinical, and VLE parameters for each specimen is available in supplemental Table 1 (see supplementary information).

\section{Signal intensity analysis}

Due to technical constraints, VLE scans from two EMR specimens (one T1a and one T1b) were excluded from the cross-sectional image analysis. When aggregated, the remaining EMR scans yielded 2,170 T1a and 2,210 T1b individual VLE images. The quantitative signal intensity analysis is displayed in - Fig. 2. In both groups, signal intensity was highest at the balloon surface and decayed rapidly over the depth of the specimen. However, the T1b scans displayed significantly higher signal intensity at every pixel of depth up to 200 pixels $(840 \mu \mathrm{m})$ deep $(P<0.0001)$.

On cross-sectional signal intensity analysis, a greater difference existed between T1a and T1b signal intensity from approximately 67 to $294 \mu \mathrm{m}$ of depth. To account for tissue heterogeneity, we constructed an en-face map of signal intensity across the specimen at $150 \mu \mathrm{m}$, the point approximating the largest difference between the curves. At $150 \mu \mathrm{m}$, mean ( \pm SD) signal intensity for T1a specimens was 109.5 AU (Arbitrary Units) ( \pm 15.5$)$ and for T1b specimens was 123.4 AU ( \pm 13.9$)$. These images were then analyzed as outlined above to identify the ROI of greatest intensity. Within this ROI, both the average signal intensity and the maximal value were calculated and results are displayed in $>$ Fig. 3 . Both average and maximal intensity were significantly higher in T1b specimens $(P<0.001)$.

\section{Signal attenuation analysis}

Results of quantitative signal attenuation analysis are displayed in $>$ Fig. 4. The mean $( \pm S D)$ signal attenuation for T1a and T1b specimens were $6.2( \pm 1.3) \mathrm{dB} / \mathrm{mm}$ and $8( \pm 2.1) \mathrm{dB} / \mathrm{mm}$, respec- 


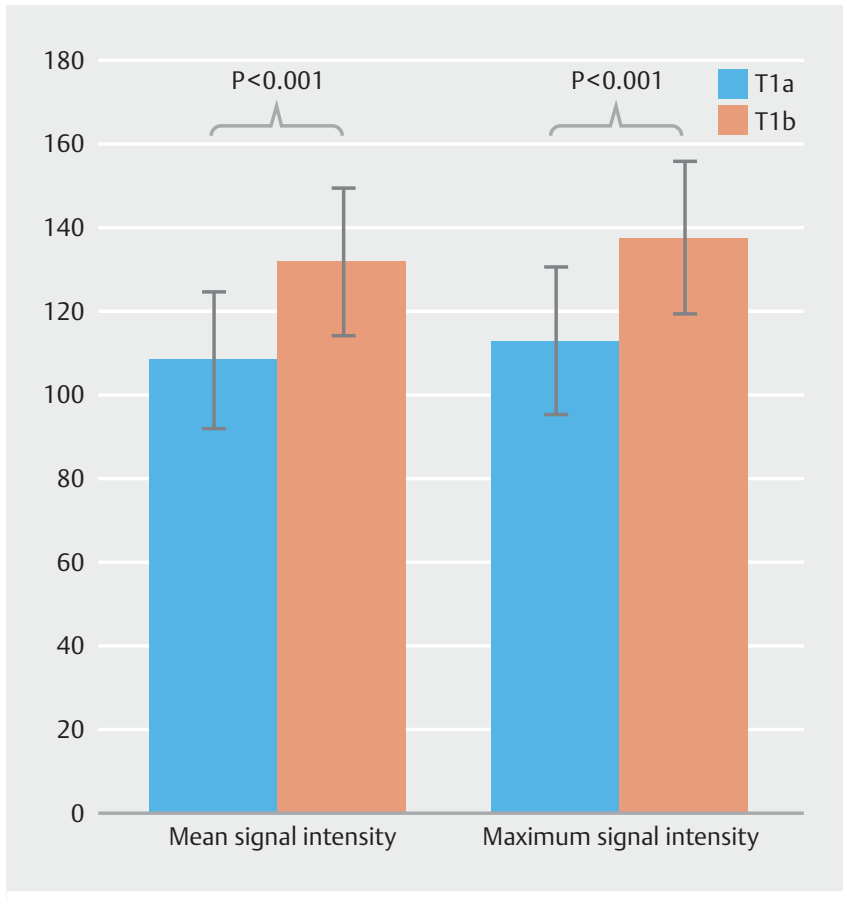

- Fig. 3 Signal intensity plots for the ROI en-face signal intensity analysis. Mean signal intensity represents the mean value of the grid box containing the highest intensity value. Maximum represents the pixel with highest signal intensity within the highest intensity grid box.

tively $(P=0.03)$. Signal attenuation values were analyzed in comparison to EAC stage to generate an optimal diagnostic threshold. Diagnostic accuracy for T1b EAC was maximized at two separate threshold values of $8 \mathrm{~dB} / \mathrm{mm}$ and $6.5 \mathrm{~dB} / \mathrm{mm}$. At $8 \mathrm{~dB} / \mathrm{mm}$, specificity was maximized at $93.3 \%(95 \% \mathrm{Cl} 82.7-$ $100 \%$ ) with a false-positive rate (i.e. T1b erroneously suggested by VLE) of only $12.5 \%$, but at a cost to sensitivity $(46.7 \%$; $05 \% \mathrm{Cl} 25.3-68 \%)$. Conversely, at $6.5 \mathrm{~dB} / \mathrm{mm}$, sensitivity was maximized at $80 \%(95 \% \mathrm{Cl} 62.9-97.1 \%)$, but at a cost to specificity (60\%; $95 \% \mathrm{Cl} 39-92 \%)$.

A logistic regression analysis of T1 EAC stage and grade of differentiation was used to estimate the predictive capacity of signal attenuation. Signal attenuation slope was associated with a significantly increased odds ratio of 1.04 for stage $(P=$ $0.034,95 \% \mathrm{Cl} 1.01-1.08)$ when controlling for grade of differentiation. These findings suggest that odds of increased stage (T1b vs. T1a) increase by $4 \%$ with every unit increase in signal attenuation slope (equivalent to each $0.06 \mathrm{~dB} / \mathrm{mm}$ ). In a logistic regression model, the bootstrap adjusted AUC for stage controlling for grade of differentiation was 0.67 (95\% Cl $0.45-$ $0.88)$.

\section{Prediction of other histologic features}

In addition to assessing the T1 EAC stage, a logistic regression analysis was conducted to assess the predictive capacity of signal attenuation for grade of differentiation (well vs. moderate/ poorly differentiated). When controlling for T1 stage, signal attenuation slope was associated with a significantly increased

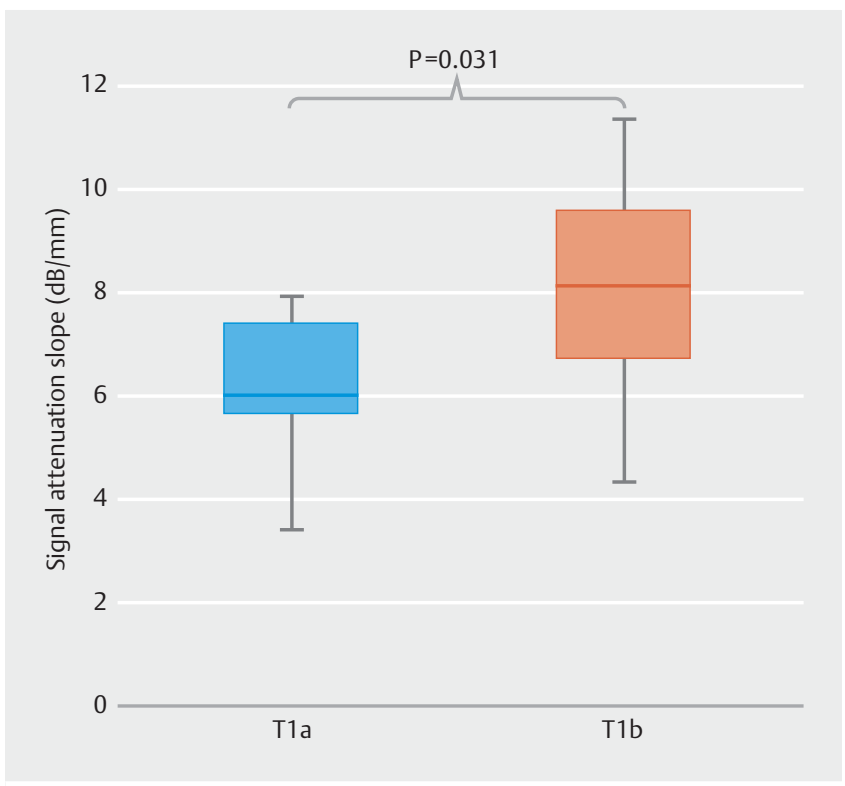

- Fig. 4 Box-and-whisker plot of signal attenuation values for T1a and T1b EMR VLE scans.

odds ratio of 1.04 for grade of differentiation ( $P=0.048$, $95 \%$ $\mathrm{Cl} 1$-1.08). Representative VLE and histologic images are shown in > Fig. 5 . Similar to the relationship to T1 stage, odds of a lower grade of differentiation increase by $4 \%$ with every unit increase in signal attenuation slope, with a bootstrap adjusted AUC of 0.68 (95\% Cl $0.47-0.91)$. Only a single patient in our cohort was found to have LVI, precluding any statistical analysis.

\section{Multifactor prediction model}

A decision-tree model was utilized to combine all collected data from both VLE and pathologic analysis. Signal attenuation slope was chosen by the model as the most informative predictor of EAC stage, followed by average signal intensity at $150 \mu \mathrm{m}$. A signal attenuation threshold of $8 \mathrm{~dB} / \mathrm{mm}$ was chosen by the model as the best predictor of EAC stage, with higher values signifying T1b and lower values T1a. An average signal intensity threshold at $150 \mu \mathrm{m}$ of $137 \mathrm{AU}$ was chosen by the model as the most informative predictor in cases where signal attenuation was less than $8 \mathrm{~dB} / \mathrm{mm}$, again with higher values signifying T1b EAC. Cases with signal attenuation $>8 \mathrm{~dB} / \mathrm{mm}$ were not further divided by the model. The model was associated with a bootstrap adjusted AUC of 0.77 (95\% Cl $0.063-0.92)$.

\section{Subset analysis}

Three specimens (2 T1a, $1 \mathrm{~T} 1 \mathrm{~b}$ ) were found to have gastric mucosa present. After excluding these samples, the remaining 27 specimens were reanalyzed. In signal attenuation analysis, mean values for $\mathrm{T} 1 \mathrm{a}$ and $\mathrm{T} 1 \mathrm{~b}$ were $6.5 \mathrm{~dB} / \mathrm{mm}( \pm 1.1)$ and $7.8 \mathrm{~dB} / \mathrm{mm}( \pm 2.1)$, respectively $(P=0.05)$. For the ROI signal intensity analysis at $150 \mu \mathrm{m}, \mathrm{T} 1 \mathrm{a}$ and $\mathrm{T} 1 \mathrm{~b}$ remained significantly different for both mean and maximum values $(P<0.001)$.

To exclude the possibility that other tissues types (e.g. nondysplastic BE, BE-LGD, BE-HGD, and squamous epithelium) 


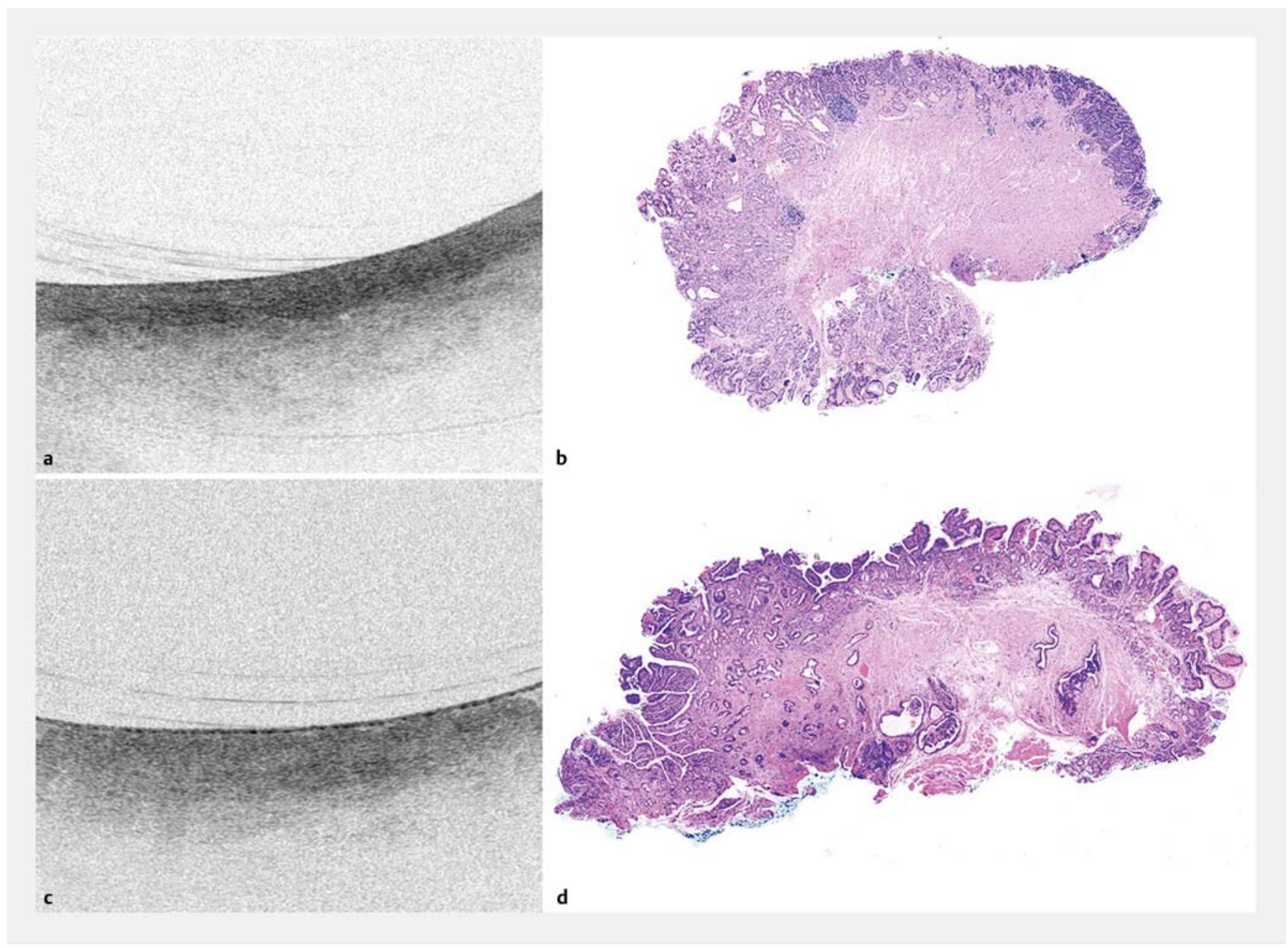

- Fig. 5 Representative VLE a, $\mathbf{c}$ and histopathology $\mathbf{b}, \mathbf{d}$ images demonstrating the relationship of signal attenuation slope to grade of differentiation. The top and bottom samples contain moderately-differentiated and well-differentiated EAC, respectively.

would affect the signal attenuation analysis, we also conducted an analysis limited to samples containing only EAC. This analysis was limited by sample size, with only $4 \mathrm{~T} 1 \mathrm{a}$ and $10 \mathrm{~T} 1 \mathrm{~b}$ specimens. However, when comparing samples with only cancerous tissue to those containing other tissue types, there was no statistically significant difference in mean signal attenuation for T1a (6.1 vs. $6.3 \mathrm{~dB} / \mathrm{mm}, P=0.81)$ and T1b (7.4 vs. $8.9 \mathrm{~dB} / \mathrm{mm}$, $P=0.17)$ groups.

\section{Discussion}

Patients with superficial EAC must be accurately staged to determine the appropriate therapeutic approach (i.e. endoscopic resection vs. esophagectomy). In this feasibility study, we used ex vivo VLE to demonstrate that $\mathrm{T} 1 \mathrm{a}$ and $\mathrm{T} 1 \mathrm{~b}$ resection specimens exhibit quantitatively distinct signal intensity and attenuation profiles. Furthermore, grade of differentiation was notably associated with VLE signal attenuation, regardless of T1 stage. This is the first study to examine use of VLE in early EAC staging and suggests that the technology may serve a role in staging T1 EAC.
While many solid tumors are staged radiographically, EAC provides a unique challenge for radiographic staging because the rate of LNM and curative resection are tied to microscopic changes in tissue invasion depth. Data on the relationship between LNM and submucosal invasion are mixed, with some advocating unacceptable risk of LNM with endoscopic resection regardless of level of submucosal invasion [16-18]. Other recent studies have demonstrated lower LNM rates, particularly in $\operatorname{sm} 1(<500 \mu \mathrm{m})$ invasion, suggesting that endoscopic resection may be advantageous both from the standpoint of avoiding surgical complications and also because of an association with lower rates of metastatic disease [19-21]. Regardless, endoscopic resection of T1b EAC is currently confined to selected patients in expert centers and is not routinely encouraged [11].

Given the critical importance of distinguishing T1a from T1b disease and the established accuracy of EUS for identifying and staging nodal disease in EAC $[22,23]$, high-frequency ultrasonographic probes were initially investigated for T-staging of superficial EAC. Several studies have demonstrated a propensity for both over and under-staging of T1 EAC, with suboptimal accuracy [24-28]. Accuracy of VLE in staging T1 EAC in the cur- 
rent study is comparable to reported accuracy of high-frequency EUS for staging EAC is $65 \%$ to $85 \%$. However, it should be noted that studies of EUS staging occurred primarily in vivo and this is an ex vivo feasibility study. Furthermore, high-frequency EUS probes differ from standard clinical echoendoscopes and are no longer routinely used for this indication. Further study of the in vivo application of the currentt model is required before a meaningful comparison can be made.

Our study is the first to demonstrate that VLE signal intensity and attenuation profiles differ quantitatively in T1a and T1b EAC. In signal intensity analysis, average signal intensity curves as a function of depth were statistically distinct throughout the entire depth of the imaged specimen. When an ROI was defined to localize the cancerous region within an EMR, signal intensity measures were also statistically distinct. Similarly, the slope of signal attenuation differed between EAC subtypes and was the feature most predictive of EAC stage.

While EAC staging is novel for VLE, OCT has been previously studied in staging of esophageal squamous cell carcinoma (SCC). Hatta and colleagues examined use of first-generation OCT for superficial SCC in over 180 patients $[29,30]$ and established criteria for predicting invasion into each esophageal layer on resection histopathology, with an overall accuracy of $90 \%$ to $92.7 \%$. Tissue composition of SCC allows for deeper penetration of the OCT laser and, hence, better delineation of the involvement of each layer. By contrast, the VLE appearance of EAC is characterized by increased surface signal intensity and a homogeneous subsurface appearance with notable lack of tissue layering. This may be due to proliferation of malignant glands and desmoplastic stroma and a tumor microenvironment that inhibits laser penetration. For this reason, we chose to analyze the VLE features of signal intensity and attenuation in our study as a proxy for T-stage. We hypothesized that deeper invasion in T1b EAC would accentuate both features and that a quantitatively higher impediment to signal penetration would be observed, as was the case.

Although T1a and T1b specimens were quantitatively distinct, the diagnostic performance of VLE in this ex vivo feasibility study was modest. When signal attenuation alone was analyzed to determine a diagnostic threshold, a clear dichotomy occurred between maximizing sensitivity and specificity. Clinically, it would be most important for a staging modality in this setting to exhibit higher specificity, so as to avoid a false-positive result. This is because a "positive" finding would indicate T1b disease and suggest caution for endoscopic resection. Further study will be needed to clarify whether sm level can be predicted by VLE parameters. By contrast, a false-negative finding is less worrisome, as the endoscopist would likely resect the presumed T1a EAC, only to find T1b staging on EMR histopathology. Our analysis yielded a maximal AUC of 0.77 at a diagnostic threshold of $8 \mathrm{~dB} / \mathrm{mm}$. While this represents a specificity of $93.3 \%$, sensitivity was only $46.7 \%$.

This is the first study to demonstrate that VLE signal attenuation is associated with grade of differentiation in T1 EAC. Although depth of invasion is a critical prognostic marker, grade of differentiation is also closely tied to the rate of LNM in T1 EAC $[7,17]$. Pathologically, grade of differentiation in EAC is classified by glandular formation in a directly proportional relationship [31]. As tumors become less differentiated, rather than glandular tissue, they are constituted by irregular, pleomorphic cells and adjacent desmoplastic stroma. These features may increasingly impede tissue penetration in VLE leading to increased signal attenuation.

This study has several important strengths, most notably its novelty and the rigor of quantitative VLE analysis performed. The majority of prior literature on VLE for the diagnosis of BErelated neoplasia is based on criteria for subjective interpretation. Although experts in VLE interpretation exhibit impressive interobserver agreement [32] for diagnosis of BE-neoplasia, these criteria have not been tested in community studies. In our study, VLE interpretation was entirely quantitative, objective, and did not incorporate any element of human decisionmaking. This is in keeping with a general trend towards quantitative analysis in VLE and development of computer-aided detection (CAD) algorithms to facilitate accessibility to the nonexpert VLE endoscopist $[33,34]$. The histological analysis was similarly rigorous, as slides were re-examined and important features confirmed.

We acknowledge several important limitations of this study. First, use of ex vivo EMR VLE scans limits the generalizability of our findings to in vivo application. Resected tissue exhibits cautery distortion artifact and rapidly undergoes cellular changes that may conceivably alter its optical properties [35]. While EMR was conducted by the same endoscopist and processed using the same protocol, all sources of possible variation could not be accounted for. EMR specimens invariably contain adjacent non-cancerous tissue which could have altered signal intensity analysis. Although we attempted to identify the region of cancer and conducted a separate ROI analysis, the precise location of EAC in an EMR cannot be definitively identified on VLE scans. We did conduct subset analyses excluding gastric mucosa and all other non-cancerous tissue types and the relationship between our measured variables was essentially preserved, with borderline statistical significance likely reflective of sample size. Presence of gastric mucosa was associated with bidirectional changes in signal intensity and attenuation, further arguing against a systematic bias. Nevertheless, future studies will need to identify a reliable method to delineate the GE junction and incorporate real-time laser marking to specify the resected region. Additionally, in all but one case, the resected EAC had been previously biopsied and the majority of these biopsies occurred within 3 months of the endoscopic resection (see supplemental Table 1). Biopsies could have induced subtle scare formation within the cancer tissue, which may have altered the optical properties. Presumably, this alteration would have occurred consistently regardless of stage, but this alteration could not be factored into the analysis. Finally, the small sample size did not allow for analysis of other important prognostic indicators, such as lymphovascular invasion and margin positivity. 


\section{Conclusion}

In conclusion, the current study quantitatively analyzed VLE scans from ex-vivo EMR specimens in T1 EAC and demonstrated differential patterns of signal intensity and signal attenuation. Our results demonstrate reasonable accuracy for noninvasive diagnosis of T1 EAC stage and grade of differentiation, both important prognostic determinants in EAC. The derived diagnostic thresholds will require further prospective testing in a validation cohort, with expected refinement of the algorithm in the in vivo setting. Although preliminary, these findings suggest that VLE may provide the combination of wide-field imaging, depth of penetration, and spatial resolution to realize the potential for noninvasive staging in superficial EAC.

\section{Competing interests}

Allon Kahn - indirect research support and an unrestricted travel grant from NinePoint Medical. Kenneth K. Wang - consultant for NinePoint Medical. Cadman L. Leggett - indirect research support from NinePoint Medical

\section{References}

[1] Hur C, Miller M, Kong CY et al. Trends in esophageal adenocarcinoma incidence and mortality. Cancer 2013; 119: 1149-1158

[2] Siegel RL, Miller KD, Jemal A. Cancer statistics, 2016. CA Cancer J Clin 2016; 66: $7-30$

[3] May A, Ell C. Diagnosis and treatment of early esophageal cancer. Curr Opin Gastroenterol 2006; 22: 433-436

[4] Stein H], Feith M, Bruecher BL et al. Early esophageal cancer: pattern of lymphatic spread and prognostic factors for long-term survival after surgical resection. Ann Surg 2005; 242: 566 - 573; discussion 573-565

[5] Das A, Singh V, Fleischer DE et al. A comparison of endoscopic treatment and surgery in early esophageal cancer: an analysis of surveillance epidemiology and end results data. Am J Gastroenterol 2008; 103: $1340-1345$

[6] Ell C, May A, Pech O et al. Curative endoscopic resection of early esophageal adenocarcinomas (Barrett's cancer). Gastrointest Endosc 2007; 65: 3-10

[7] Leggett CL, Lewis JT, Wu TT et al. Clinical and histologic determinants of mortality for patients with Barrett's esophagus-related T1 esophageal adenocarcinoma. Clin Gastroenterol Hepatol 2015; 13: 658 - 664 e651-653

[8] Nijhawan PK, Wang KK. Endoscopic mucosal resection for lesions with endoscopic features suggestive of malignancy and high-grade dysplasia within Barrett's esophagus. Gastrointest Endosc 2000; 52: $328-332$

[9] Hull M], Mino-Kenudson M, Nishioka NS et al. Endoscopic mucosal resection: an improved diagnostic procedure for early gastroesophageal epithelial neoplasms. Am J Surg Pathol 2006; 30: 114-118

[10] Namasivayam V, Wang KK, Prasad GA. Endoscopic mucosal resection in the management of esophageal neoplasia: current status and future directions. Clin Gastroenterol Hepatol 2010; 8: 743-754; quiz e796
[11] Shaheen NJ, Falk GW, lyer PG et al. ACG Clinical Guideline: Diagnosis and Management of Barrett's Esophagus. Am J Gastroenterol 2016; 111: $30-50$

[12] Evans JA, Bouma BE, Bressner J et al. Identifying intestinal metaplasia at the squamocolumnar junction by using optical coherence tomography. Gastrointest Endosc 2007; 65: 50 - 56

[13] Leggett CL, Gorospe EC, Chan DK et al. Comparative diagnostic performance of volumetric laser endomicroscopy and confocal laser endomicroscopy in the detection of dysplasia associated with Barrett's esophagus. Gastrointest Endosc 2016; 83: 880 - 888 e882

[14] Kohli DR, Schubert ML, Zfass AM et al. Performance characteristics of optical coherence tomography in assessment of Barrett's esophagus and esophageal cancer: systematic review. Dis Esophagus 2017; 30 : $1-8$

[15] Schindelin J, Arganda-Carreras I, Frise E et al. Fiji: an open-source platform for biological-image analysis. Nat Methods 2012; 9: 676682

[16] Badreddine RJ, Prasad GA, Lewis JT et al. Depth of submucosal invasion does not predict lymph node metastasis and survival of patients with esophageal carcinoma. Clin Gastroenterol Hepatol 2010; 8: $248-253$

[17] Leers JM, DeMeester SR, Oezcelik A et al. The prevalence of lymph node metastases in patients with $\mathrm{T} 1$ esophageal adenocarcinoma a retrospective review of esophagectomy specimens. Ann Surg 2011; 253: $271-278$

[18] Rice TW, Zuccaro G Jr, Adelstein DJ et al. Esophageal carcinoma: depth of tumor invasion is predictive of regional lymph node status. Ann Thorac Surg 1998; 65: 787-792

[19] Manner H, May A, Pech O et al. Early Barrett's carcinoma with “lowrisk" submucosal invasion: long-term results of endoscopic resection with a curative intent. Am J Gastroenterol 2008; 103: 2589-2597

[20] Manner $\mathrm{H}$, Pech O, Heldmann $\mathrm{Y}$ et al. Efficacy, safety, and long-term results of endoscopic treatment for early stage adenocarcinoma of the esophagus with low-risk sm 1 invasion. Clin Gastroenterol Hepatol 2013; 11: 630-635; quiz e645

[21] Scholvinck D, Kunzli H, Meijer $S$ et al. Management of patients with T1b esophageal adenocarcinoma: a retrospective cohort study on patient management and risk of metastatic disease. Surg Endosc 2016; 30: $4102-4113$

[22] Chandawarkar RY, Kakegawa T, Fujita $\mathrm{H}$ et al. Endosonography for preoperative staging of specific nodal groups associated with esophageal cancer. World J Surg 1996; 20: 700 - 702

[23] Rosch T. Endosonographic staging of esophageal cancer: a review of literature results. Gastrointest Endosc Clin N Am 1995; 5: 537- 547

[24] Bergeron EJ, Lin J, Chang AC et al. Endoscopic ultrasound is inadequate to determine which T1/T2 esophageal tumors are candidates for endoluminal therapies. J Thorac Cardiovasc Surg 2014; 147: 765 771, discussion 771-763

[25] Bulsiewicz W], Dellon ES, Rogers AJ et al. The impact of endoscopic ultrasound findings on clinical decision making in Barrett's esophagus with high-grade dysplasia or early esophageal adenocarcinoma. Dis Esophagus 2014; 27: 409-417

[26] Pouw RE, Heldoorn N, Alvarez Herrero L et al. Do we still need EUS in the workup of patients with early esophageal neoplasia? A retrospective analysis of 131 cases Gastrointest Endosc 2011; 73: 662-668

[27] Heidemann J, Schilling MK, Schmassmann A et al. Accuracy of endoscopic ultrasonography in preoperative staging of esophageal carcinoma. Dig Surg 2000; 17: 219-224

[28] Waxman I, Raju GS, Critchlow J et al. High-frequency probe ultrasonography has limited accuracy for detecting invasive adenocarcinoma in patients with Barrett's esophagus and high-grade dysplasia or intramucosal carcinoma: a case series. Am J Gastroenterol 2006; 101: $1773-1779$ 
[29] Hatta W, Uno K, Koike T et al. A prospective comparative study of optical coherence tomography and EUS for tumor staging of superficial esophageal squamous cell carcinoma. Gastrointest Endosc 2012; 76: $548-555$

[30] Hatta W, Uno K, Koike T et al. Optical coherence tomography for the staging of tumor infiltration in superficial esophageal squamous cell carcinoma. Gastrointest Endosc 2010; 71: 899-906

[31] Matkovic E, Schwalbe M, Matkowskyj KA. Pathologic features of esophageal and gastric malignancies. Cancer Treat Res 2016; 168: $17-43$

[32] Trindade AJ, Inamdar S, Smith MS et al. Volumetric laser endomicroscopy in Barrett's esophagus: interobserver agreement for interpre- tation of Barrett's esophagus and associated neoplasia among highfrequency users. Gastrointest Endosc 2017; 86: 133 - 139

[33] Swager AF, van der Sommen F, Klomp SR et al. Computer-aided detection of early Barrett's neoplasia using volumetric laser endomicroscopy. Gastrointest Endosc 2017; 86: 839-846

[34] Kahn A, Pai RK, Fukami N. Novel computer-enhanced visualization of volumetric laser endomicroscopy correlates endoscopic and pathological images. Clin Gastroenterol Hepatol 2018; 16: A23 - A24

[35] Bejarano PA, Berho M. Examination of surgical specimens of the esophagus. Arch Pathol Lab Med 2015; 139: 1446 - 1454 